\title{
Robust Reconfiguration of A Distribution System
}

\author{
Benyamin Moradzadeh*, Guodong Liu ${ }^{\dagger}$ and Kevin Tomsovic ${ }^{\ddagger}$ \\ *Integrated Resource Planning, Pacific Gas and Electric Company \\ San Francisco, California 94105 \\ Email: Benyamin.Moradzadeh@pge.com \\ ${ }^{\dagger}$ Power and Energy Systems Group, Oak Ridge National Laboratory \\ Oak Ridge, Tennessee 37381 \\ Email: liug@ornl.gov \\ ${ }^{\ddagger}$ Department of Electrical Engineering and Computer Science \\ University of Tennessee, Knoxville, Tennessee 37996 \\ Email: tomsovic@tennessee.edu
}

\begin{abstract}
In this paper, a robust reconfiguration approach based on Mixed Integer Programming (MIP) is proposed to minimize loss in distribution systems. A Depth-First Search (DFS) algorithm to enumerate possible loops provides radiality constraint. This provides a general solution to the radiality constraint for distribution system reconfiguration/expansion problems. Still, imprecision and ambiguity in net loads, i.e. load minus renewable generation, due to lack of sufficient measurements and high utilization of demand response programs and renewable resources, creates challenges for effective reconfiguration. Deterministic optimization of reconfiguration may no lead to optimal/feasible results. Two methods to address these uncertainties are introduced in this paper: one, based on a stochastic MIP (SMIP) formulation and two, based on a fuzzy MIP (FMIP) formulation. Case studies demonstrate the robustness and efficiency of the proposed reconfiguration methods.
\end{abstract}

Keywords-Distribution System Reconfiguration (DSR), Depth-First Search (DFS), Fuzzy Mixed Integer Programming (FMIP), Stochastic Mixed Integer Programming (SMIP).

\section{NOMENCLATURE}

$\begin{array}{ll}P_{j}^{f}, Q_{j}^{f} & \text { Active and reactive power flow on branch } j . \\ P_{i}^{b}, Q_{i}^{b} & \begin{array}{l}\text { Active and reactive power output of the bat- } \\ \text { tery on bus } i .\end{array} \\ X_{j} & \text { Status of the switch on line } j(0: \text { off, } 1 \text { : on) } \\ r_{j}, x_{j} & \text { Resistance and reactance of line } j .\end{array}$

This manuscript has been authored by UT-Battelle, LLC under Contract No. DE-AC05-00OR22725 with the U.S. Department of Energy. The United States Government retains and the publisher, by accepting the article for publication, acknowledges that the United States Government retains a nonexclusive, paid-up, irrevocable, world-wide license to publish or reproduce the published form of this manuscript, or allow others to do so, for United States Government purposes. The Department of Energy will provide public access to these results of federally sponsored research in accordance with the DOE Public Access Plan(http://energy.gov/downloads/doe-publicaccess-plan).

This work also made use of Engineering Research Center Shared Facilities supported by the Engineering Research Center Program of the National Science Foundation and the Department of Energy under NSF Award Number EEC-1041877 and the CURENT Industry Partnership Program.
$P^{f}, Q^{f} \quad$ Vectors of active and reactive power flow on lines.

$\boldsymbol{P}, \boldsymbol{Q}$ Vectors of active and reactive power net loads.

$P^{b}, Q^{b} \quad$ Active and reactive power output of battery units.

$I_{j} \quad$ Current on line $j$.

$I_{j}^{\max } \quad$ Thermal limit of line $j$.

$V_{i} \quad$ Voltage magnitude on node $i$.

$V_{i}^{\max } \quad$ Maximum acceptable voltage magnitude on node $i$.

$V_{i}^{\min } \quad$ Minimum acceptable voltage magnitude on node $i$.

$\Delta V_{\max } \quad$ Maximum allowed voltage deviation from substation voltage level.

$\alpha_{k}^{i} \quad k^{t h}$ path reaching bus $i$ from substation $s$.

$N_{k}^{i} \quad$ Number of switches in path $\alpha_{k}^{i}$.

$N_{l} \quad$ Number of switches in loop $l$.

$\Delta \boldsymbol{P}, \boldsymbol{\Delta Q}$ Maximum variation of active and reactive power load.

$\boldsymbol{A} \quad$ Reduced node incidence matrix.

$P_{\max }^{c h}, P_{\max }^{d c}$ Maximum charge and discharge rates of a battery.

$P F_{i}^{b, \min }$ Minimum acceptable power factor of the battery storage unit $i$.

$S C_{i}^{b}, C_{i}^{b} \quad$ State of charge and capacity of the battery storage unit $i$.

$d_{i}^{b} \quad$ Acceptable depth of discharge of battery $i$.

$t \quad$ Charging or discharging time.

$n_{j} \quad$ Number of lines in system.

$n_{b} \quad$ Number of batteries in the system.

$n_{\omega} \quad$ Number of scenarios used for stochastic optimization.

$\omega \quad$ Index of load scenario in the stochastic optimization.

$\pi_{\omega} \quad$ Probability of scenario $\omega$ in the stochastic optimization. 


\author{
Membership function in the fuzzy optimiza- \\ tion. \\ Index of branches. \\ Index of buses. \\ Index of loops/meshes.
}

\section{INTRODUCTION}

$\mathbf{R}$ ECONFIGURATION is used worldwide as the preferred technique to reduce power loss [1]- [14], restoreout-of-service zones, and decrease network overloading by altering the topological structures of distribution feeders [15]. However, optimization of DSR is incredibly difficult due to the structures of the distribution networks which are mainly radial and include open and closed switches whose status would be controlled to achieve the aforementioned desired outcomes. If exact optimization techniques such as MIP are employed, the radiality constraints must be explicitly represented in the mathematical modeling. This is not the case, however, when heuristic or meta-heuristic techniques are used, where the radiality constraints are controlled implicitly [16]. Nevertheless, these solutions suffer from a number of shortcomings, including scalability, and the possibility of a suboptimal solution and poor convergence.

Another problem that exists in DSR is because of high level of uncertainty in net loads as a direct result of poor measurements and increasing penetration of controllable loads and renewable resources. Therefore, deterministic optimization of DSR may not lead to optimal or even feasible results which respect the operational constraints.

A modified linear programming formulation is proposed in [1], to minimize loss through penalizing high power flows on the lines with high resistance. The drawback in this method is that the linear formulation of the loss minimization problem only gives the sub-optimal solution. An optimal power flow (OPF)-based DSR using MIP is presented in [2]. However, the formulation does not guarantee radiality.

Different heuristic algorithms are presented in [3]-[6] to solve the DSR problem. The process begins with a completely meshed network. In each step of the heuristic algorithm, a branch is opened to maintain a radial topology. These algorithms apply different sensitivity analyses to perform switching operations in each iteration.

Population-based optimization algorithms are used in [7] and [12] to find the optimal configuration with minimum loss. Reference [13] employs a simulated annealing metaheuristic technique for loss minimization-based DSR. This work expands on these prior efforts to address system uncertainties.

Reference [17] proposes a reliability-oriented reconfiguration method that includes load uncertainties. The method uses interval analysis to quantify the impact of uncertain data and to maximize the possibility of reliability improvement and/or loss reduction. A procedure for supply restoration in distribution networks based on fuzzy risk management is proposed in [18]. The method models uncertainty in recognizing consumer loads by describing them with fuzzy numbers. The approach proposed in [19] considers both system reliability and loss in the reconfiguration. A clonal selection algorithm is used to deal with uncertainty of the random parameters.

The net load uncertainty has been rarely considered in the DSR papers. As mentioned before, a feasible/optimal reconfiguration cannot be guaranteed by deterministic approaches. In this paper, a general approach based on MIP is proposed to formulate DSR in the presence of load uncertainty. This study considers distributed battery units in the DSR problem. This is because of the increasing attention to these devices which is mainly due to their performance in reducing equipment loading during peak hours and helping with renewable energy resource integration [20].

SMIP and FMIP are used to obtain optimal solutions which are robust under different net loads. The study shows that relatively minor increase in robustness from SMIP over FMIP requires far more computation. This suggests that the developed FMIP approach would be preferred for realtime applications. Hereafter in this paper the word "load" represents "net load".

\section{Problem Formulation}

In this section, a deterministic MIP formulation of DSR is described. The proposed FMIP and SMIP formulations are then presented to solve the reconfiguration problem with load uncertainty.

\section{A. MIP-Based DSR}

The DSR optimization problem considered in this study is given as the minimization of active power loss subject to a set of constraints including power balance, thermal limits of the lines, voltage limit, radiality of the system, and battery units' constraints. Specifically,

$$
\min \sum_{j=1}^{n_{j}} r_{j}\left[\left(P_{j}^{f}\right)^{2}+\left(Q_{i}^{f}\right)^{2}\right]+\sum_{i=1}^{n_{b}}\left(P_{i}^{b}\right)^{2}
$$

Subject to:

- Power flow constraints

$$
\begin{aligned}
& A^{T} P^{f}=P-P^{b} \\
& A^{T} Q^{f}=Q-Q^{b}
\end{aligned}
$$

- Line thermal limit constraint

$$
I_{j} \leq X_{j} I_{j}^{\max }
$$

- Voltage constraint

$$
V_{i}^{\min } \leq V_{i} \leq V_{i}^{\max }
$$


- Radiality constraint

$$
\sum_{j=1}^{N_{l}} X_{j} \leq N_{l}-1 \quad, \forall l
$$

- Battery constraint

$$
\begin{aligned}
-\left(1-S C_{i}^{b}\right) C_{i}^{b} & \leq P_{i}^{b} t \leq C_{i}^{b} S C_{i}^{b}-d_{i}^{b} \\
-P_{\max }^{c h} & \leq P_{i}^{b} \leq P_{\max }^{d c} \\
P F_{i}^{b} & >P F_{i}^{b, \min }
\end{aligned}
$$

$$
X_{j} \in\{0,1\}
$$

where $X_{j}=0$ and $X_{j}=1$ represent an open switch and closed switch, respectively. The first term in the objective function minimizes system active power loss while the second term captures the damaging effect of fast charging and discharging of the battery units to prolong the battery life. It is worth noting that the formulation of active power loss assumes the voltage magnitude to be one per-unit.

Constraints (2)-(3) are active and reactive power balance equations. The thermal limit constraint of distribution lines and cables is guaranteed by (4). Radiality of the reconfigured system is preserved by satisfying (6). The radiality constraint is formulated based on a cycle detection approach which is separate from the optimization. In this algorithm it is assumed that all the switches in the system are closed. Then, a DFS-based algorithm is employed to detect all the cycles and loops in the system [15]. The radiality constraint (6) guarantees that there is at least one open switch in any possible loop, $l$. Voltage limits are provided by (5). Charging/discharging rate constraints of the battery units are formulated in (7)-(9). According to (9) the battery units should operate with a power factor which is higher than a specified threshold, $P F_{i}^{b, \min }$.

A linear formulation of the voltage constraint based on deviation of the voltage on a node from substation voltage is used since the key is assessing impact of a particular configuration on the voltage profile and the precise voltage is not typically needed. As shown in [21], voltage constraint for every node can be formulated by a linear formulation based on active and reactive power flows on the lines reaching the node from the substation, $s$ :

$$
2 \sum_{j \in \alpha_{k}^{i}}\left(r_{j} P_{j}+x_{j} Q_{j}\right) \leq \delta_{\max }, \forall i
$$

where $\delta_{\max }=\Delta V_{\max }\left(2 V_{s}-\Delta V_{\max }\right)$. Modification to (10) needs to be made in order to prevent the constraint from being binding for any inactive path corresponding to bus $j$ (an inactive path is a path from substation to the node in which there is one or more open branches). Therefore, an additional term is added to the right hand side of (10) as follows:

$$
2 \sum_{j \in \alpha_{k}^{i}}\left(r_{j} P_{j}+x_{j} Q_{j}\right) \leq \delta_{\max }+M\left(N_{k}^{i}-\sum_{j \in \alpha_{k}^{i}} X_{j}\right), \forall i
$$

where $M$ is an arbitrary large positive number, e.g. 1E10.

\section{B. Reconfiguration with Load Uncertainty}

Load uncertainties affect both feasibility and optimality. In this paper, two different approaches are proposed to formulate the reconfiguration problem with uncertain data. In the first approach, DSR is formulated as a stochastic optimization problem based on load probability distribution functions (PDF). The second approach is based on a FMIP in which the objective function and constraints are converted to fuzzy or interval relations. This means that the constraints are allowed to be violated with some margin as defined by their membership functions. The goal is to compare the two algorithms in terms of speed and robustness. Speed is particularly important for objectives such as system restoration after faults.

1) SMIP-Based DSR: The traditional two-stage integer programming approach is used to solve the SMIP-based DSR. Status of the switches and batteries output power are first stage variables while power flows on the lines and voltage magnitude on the nodes are considered as the second stage variables. This formulation makes sure that the obtained topology and scheduled battery outputs are feasible for various scenarios.

The two-stage SMIP model is formulated as:

$\min \sum_{\omega=1}^{n_{\omega}} \pi_{\omega}\left[\sum_{j=1}^{n_{j}} r_{j}\left[\left(P_{j \omega}^{f}\right)^{2}+\left(Q_{j \omega}^{f}\right)^{2}\right]+\sum_{i=1}^{n_{b}}\left(P_{i}^{b}\right)^{2}\right]$

Subject to:

- Power flow constraints

$$
\begin{aligned}
& A^{T} P_{\omega}^{f}=P_{\omega}-P^{b} \\
& A^{T} Q_{\omega}^{f}=Q_{\omega}-Q^{b}
\end{aligned}
$$

- Line thermal limit constrain

$$
I_{j \omega} \leq X_{j} I_{j}^{\max }
$$

- Voltage constraint

$$
V_{i}^{\text {min }} \leq V_{i \omega} \leq V_{i}^{\max }
$$

- Radiality constraint 


$$
\sum_{j=1}^{N_{l}} X_{j} \leq N_{l}-1 \quad, \forall l
$$

- Battery constraints

$$
\begin{aligned}
-\left(1-S C_{i}^{b}\right) C_{i}^{b} & \leq P_{i}^{b} t \leq C_{i}^{b} S C_{i}^{b}-d_{i}^{b} \\
-P_{\max }^{c h} & \leq P_{i}^{b} \leq P_{\max }^{d c} \\
P F & >P F_{\min }
\end{aligned}
$$

$$
X_{j} \epsilon\{0,1\}
$$

An appropriate scenario set is critical to the SMIP model. Traditionally, in power system research studies, the foretasted load is modeled as a Gaussian probability distribution function with nominal load as the mean value and a standard deviation [22]-[25]. Then, Monte Carlo method is used to generate a discrete scenario set, which approximates the continuous distribution function of the foretasted load. Although the uncertainty model of the foretasted load will be more precise with higher number of scenarios, it increases the computational intensity of the problem. Therefore, an effective scenario reduction method is essential for solving large scale problems. Efficient algorithms based on backward and fast forward methods have been developed to determine the optimal scenario set.

In this paper, Monte Carlo algorithm is used to produce discrete scenarios based on Gaussian probability functions considered for the loads. The backward reduction described in [25] is used to efficiently reduce the number of scenarios.

2) FMIP-Based DSR: Since stochastic optimization approach is time consuming, an approximation of the uncertainties based on linear intervals or possibility ranges is introduced. Specifically, we assume that the load forecast is an interval number of the form:

$$
\begin{gathered}
\mathbf{P}_{l} \epsilon[\mathbf{P}-\triangle \mathbf{P}, \mathbf{P}+\triangle \mathbf{P}] \\
\mathbf{Q}_{l} \epsilon[\mathbf{Q}-\triangle \mathbf{Q}, \mathbf{Q}+\triangle \mathbf{Q}]
\end{gathered}
$$

In order to convert the deterministic optimization problem (1)-(9) into a fuzzy optimization, the power balance constraint (2)-(3), line flow limits (4), and voltage limits (11) constraints can be expressed as soft relations. Different membership functions are developed for the above constraints and objective function which are described in the following.

Power flow constraints: The optimum configuration should be able to tolerate severe loading scenarios. Because of the radial structure of distribution systems if load on every node increases, the line flows increase and there will be a higher possibility of violation in line flow and voltage limit constraints. The membership function considered for fuzzy active power balance constraints is depicted in Fig. 1. A constant power factor is considered for loads on every bus. The membership function, $\mu_{p}$ indicates that it is more desirable if power balance equations are satisfied for maximum loading cases. Although this is a conservative approach, the obtained results will be feasible for a wider range of loading scenarios.

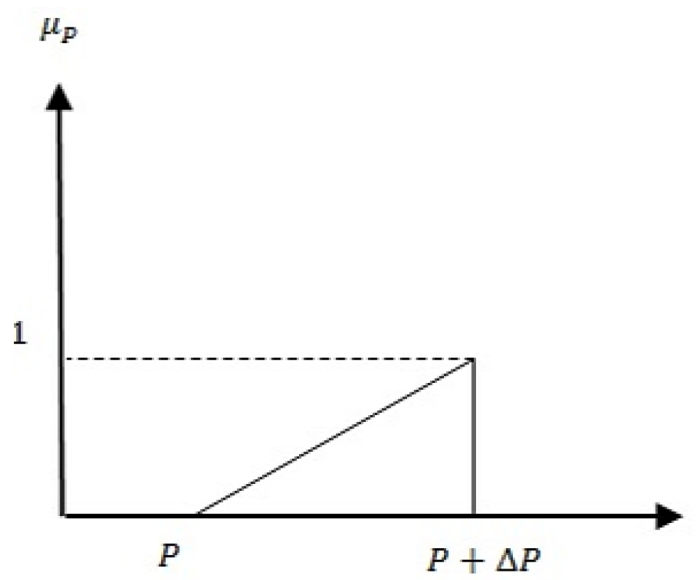

Figure 1. Membership function of the active power balance constraint

Line thermal limit constraints: Line flow limit constraint can also be modeled by a soft constraint. The membership function considered for this constraint is shown in Fig. 2. $\mu_{I}$ indicates that the configuration becomes less acceptable as the line power flows violate the thermal limits.

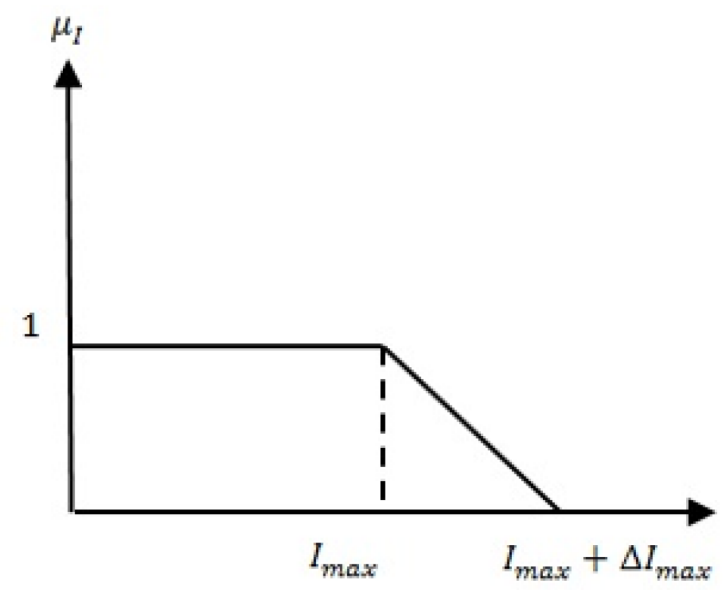

Figure 2. Membership function of line thermal limit constraint

Voltage limit constraints: Voltage limits (11) are also converted to a soft constraint to account for load uncertainty. The membership function for voltage constraint is similar to the one assigned for line thermal limit.

Based on the symmetric approach, the objective function (1), should be essentially smaller than or equal to some aspiration level, $Z_{0}$. Membership function for the objective function, $\mu_{Z}$, can be modeled by Fig. 3. The aspiration level 
represents the ideal system configuration with minimum loss and optimal battery output. A configuration becomes less acceptable as the system loss increases above the ideal value as indicated by the reduced membership in Fig. 3. One good candidate for $Z_{0}$ is the result of optimization problem (1) with nominal load values.

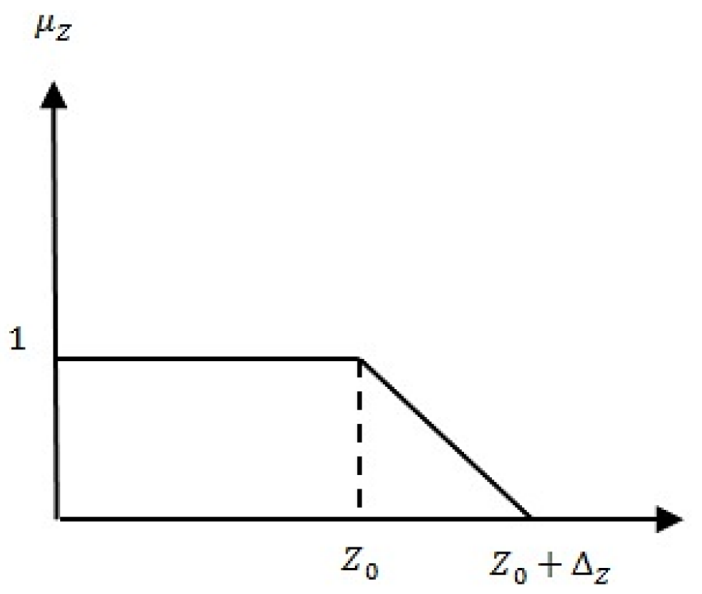

Figure 3. Membership function of the objective function

Since the constraints and objective function are represented by membership functions, min-max method can be used to solve the optimization. This is given by:

$$
\lambda^{*}=\max \min \left(\mu_{Z}, \mu_{P}, \mu_{I}, \mu_{V}\right)
$$

Consequently, the following equivalent parametric model can be used:

$$
\begin{gathered}
\max \lambda \\
\text { s.t. } \\
\mu_{Z}, \mu_{P}, \mu_{V}, \mu_{I} \geq \lambda \\
(6)-(9) \\
0 \leq \lambda \leq 1, X_{j} \epsilon\{0,1\}
\end{gathered}
$$

The problem reduces to maximizing a scaler value $\lambda$ that represents the least satisfied constraint or objective function. In the solution, all membership values are greater than or equal to this $\lambda$.

Two cases for the FMIP-based reconfiguration are considered in this study. The first case which is called FMIP with soft constraints (SC FMIP) allows the operational constraints (4) and (11) to violate with some degree based on their membership functions. In other words, $\triangle I_{\max }$ and $\triangle V_{\max }$ are non-zero values. These values can be determined by the operator depending on the desired robustness level of the solution. In the second case, FMIP with hard constraints (HC FMIP), no violation in the thermal limit and voltage constraint is allowed. Obviously, HC FMIP is a more conservative approach which allows for less flexibility in the constraints.

\section{Simulation Results}

Simulations are performed on a $2.66 \mathrm{GHz}, 4$ GB RAM PC. The software tools used to solve the MIP problems are MATLAB 2011b and ILOG CPLEX 12.2 under a 64-bit operating system. Two different electrical systems are studied to show the effectiveness of the proposed approaches.

\section{A. 32-Bus Test System}

The first system which is depicted in Fig. 4 is the 37bracnh test system used in [14]. The dashed lines represent open lines in the original configuration (normally open switches). Five battery units are located on buses 9, 17, 18, 22 , and 30 . The batteries capacity, maximum discharge rate, and minimum power factor are $75 \mathrm{~kW}, 25 \mathrm{~kW}$, and 0.9 , respectively. It is also assumed that the batteries are full before the reconfiguration process.

Before starting the optimization, the DFS-based cycle detection approach is performed on the adjacency matrix of the system graph assuming that all the switches are closed, which finds 26 cycles. The branches making different cycles are then identified and according to (6), there will be at least one open switch in each detected loop. For example, $2,3,4,5,6,7,8,21,20,19,2$ is a sequence of vertexes which creates one potential loop. Number of switches in this cycle, $N_{l}$, is 10 . Since $X_{j}=1$ represents a close switch, $\sum_{j=1}^{10} X_{j} \leq 9$ constraint avoids this loop in the reconfiguration process.

1) Deterministic DSR: In the deterministic optimization, we assume no uncertainty in the loads. The nominal loads in [14] are considered as loads in this optimization.

Table I and Table II show the result obtained by the deterministic reconfiguration. As shown in the tables, the new topology obtained by MIP-based DSR reduces system loss by $31 \%$. Obviously, injections from battery units reduces the system loss even more. Reference [15] discusses that the distributed storage units can also change the resulted configuration through changing the line flows and voltage levels (constraints (4) and (11)).

2) DSR with load uncertainty: SMIP and FMIP approaches are now applied to solve the reconfiguration problem in the presence of load uncertainty. For SMIP reconfiguration, the foretasted load is modeled as a Gaussian probability distribution function with nominal load in [14] as the mean value and standard deviation, $\sigma$, of $6 \%$. It is assumed that the load changes in the range of $P \pm 3.5 \sigma$. Monte Carlo simulation is used to generate 5000 scenarios. Number of the scenarios is reduced to 200 using the backward reduction technique mentioned in section II-B.

For FMIP, it is assumed that the load varies in the range $[\mathbf{P}-\boldsymbol{\Delta} \mathbf{P}, \mathbf{P}+\boldsymbol{\Delta} \mathbf{P}]$ where $\mathbf{P}$ is the nominal load 


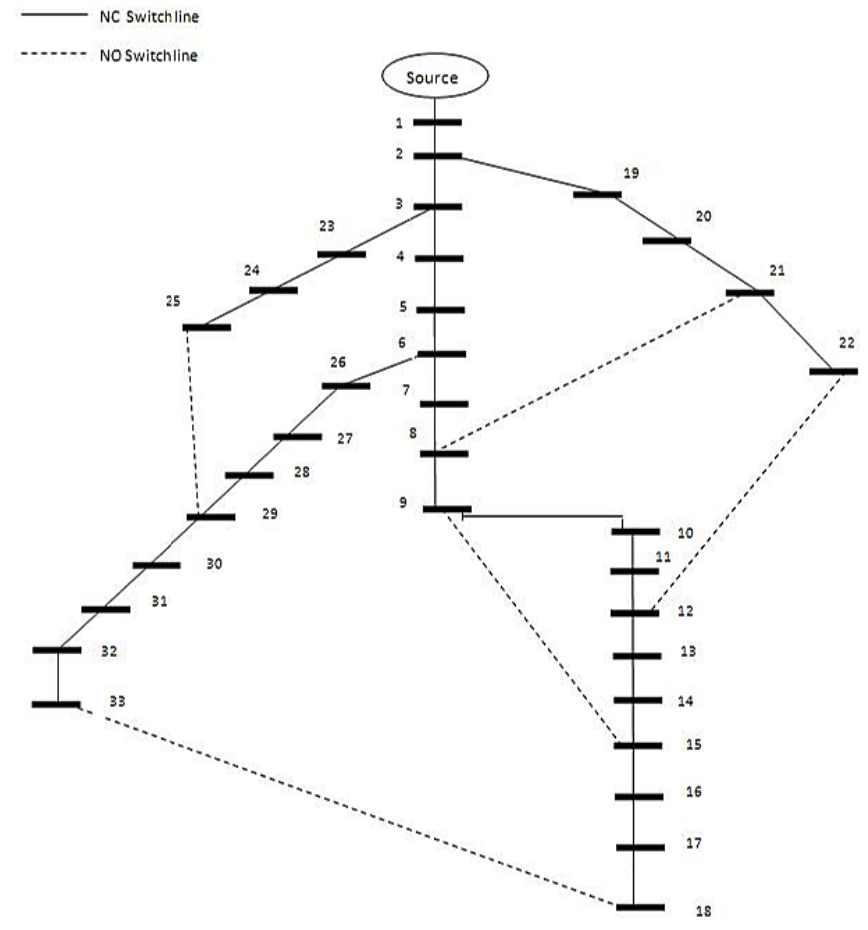

Figure 4: 32-bus test system

TABLE I

DETERMINISTIC RECONFIGURATION 32-BUS SYSTEM

\begin{tabular}{|c|c|}
\hline Parameter & MIQP \\
\hline Optimal solution- & $\begin{array}{c}7-8,9-10, \\
14-15, \\
25-29, \\
\text { open switches }\end{array}$ \\
\hline $\begin{array}{c}\text { Loss- new } \\
\text { configuration, } \\
\text { without battery (kW) }\end{array}$ & 139.55 \\
\hline $\begin{array}{c}\text { Loss- new } \\
\text { configuration, with } \\
\text { battery (kW) }\end{array}$ & 131.42 \\
\hline $\begin{array}{c}\text { Loss- original } \\
\text { configuration }(\mathrm{kW})\end{array}$ & 202.5 \\
\hline
\end{tabular}

TABLE II

BATTERY SCHEDULED POWER 32-BUS SYSTEM

\begin{tabular}{|c|c|}
\hline Bus & $\mathbf{k W}$ \\
\hline 9 & 15.63 \\
\hline 17 & 11.53 \\
\hline 18 & 19.28 \\
\hline 22 & 19.66 \\
\hline 30 & 23.89 \\
\hline
\end{tabular}

in [14] and $\Delta \mathbf{P}$ is assumed to be $21 \%$ of the nominal load as an example of large uncertainty (The load variation is in the same range for SMIP and FMIP). A constant power factor is considered for the loads on every node. As mentioned in section II-B, two cases for the FMIPbased reconfiguration are considered: SC FMIP, in which the operational constraints (4) and (11) are allowed to violate up to $10 \%\left(\triangle I_{\max }=1.1 I_{\max }\right.$ and $\left.\triangle V_{\max }=1.1 V_{\max }\right)$, and HC FMIP which does not allow for any constraint violations $\left(\triangle I_{\max }=\triangle V_{\max }=0\right)$.

Table III and Table IV show the reconfiguration result obtained by SMIP and FMIP. The third row in Table III shows the active power loss for the configurations and batteries output power obtained by different algorithms when the system nominal load is considered. As observed in Table , both FMIP and SMIP lead to configurations with higher losses compared to the deterministic case. This is because these approaches seek conservative solutions that are feasible for many different load conditions.

The configurations and scheduled output power from the batteries calculated by the deterministic MIP, SC FMIP, HC FMIP, and SMIP are evaluated for the full 5000 scenarios to to identify the percentage of scenarios leading to constraint violations. This shows the robustness level of the different solutions against variations of loads. As observed in Table $\mathrm{V}$, although HC FMIP and SMIP lead to a similar robustness level, HC FMIP is much faster (HC FMIP leads to a slightly more conservative solution relying on more power extraction from battery units). It is worth noting that because of the radial structure of distribution systems, higher loads directly lead to higher flows on the lines. Therefore, line flow limit and voltage limit constraints are more likely to be violated. This feature is considered in the membership function considered for the power balance constraints and that is the reason that FMIP leads to a reasonably robust result with such reduced computation.

TABLE III
\begin{tabular}{|c|c|c|c|}
\multicolumn{4}{c|}{ RECONFIGURATION WITH UNCERTAINTY } \\
32-BUS SYSTEM \\
\hline & SC FMIP & HC FMIP & SMIP \\
\hline & $7-8,11-$ & $7-8,11-$ & $7-8,11-$ \\
Optimal solution- & $12,14-$ & $12,14-$ & $12,14-$ \\
open switches & $15,18-$ & $15,17-$ & $15,17-$ \\
& $33,25-29$ & $18,25-29$ & $18,25-29$ \\
\hline loss & 134.47 & 138.30 & 138.94 \\
\hline
\end{tabular}

\section{B. 86-bus Test System}

The second test system, depicted in Fig. 5, contains 86 load buses, three supply buses, and 96 branches [3]. Only 28 branches are equipped with switches. The lines with blank boxes show normally open switches. The system includes 9 battery storage units on buses 23, 28, 51, 52, 63, 70. 76, 81, and 87. The batteries' capacity and maximum 
TABLE IV

BATTERY DISCHARGE WITH UNCERTAINTY 32-BUS SYSTEM

\begin{tabular}{|c|c|c|c|}
\hline & SC FMIP & HC FMIP & SMIP \\
\hline Bus & \multicolumn{3}{|c|}{ kW } \\
\hline 9 & 18.71 & 16.99 & 15.02 \\
\hline 17 & 12.69 & 12.18 & 10.76 \\
\hline 18 & 22.56 & 19.87 & 17.54 \\
\hline 22 & 22.96 & 25 & 24.84 \\
\hline 30 & 24.87 & 25 & 22.90 \\
\hline
\end{tabular}

TABLE V

COMPARISON OF THE APPROACHES

32-BUS SYSTEM

\begin{tabular}{|c|c|c|c|c|}
\hline & Deterministic & SC FMIP & HC FMIP & SMIP \\
\hline $\begin{array}{c}\text { Infeasible } \\
\text { scenarios } \\
(\%)\end{array}$ & 54 & 20.2 & 4.49 & 4.52 \\
\hline $\begin{array}{c}\text { Computation } \\
\text { time (s) }\end{array}$ & 0.5 & \multicolumn{2}{|c|}{1.32} & 49 \\
\hline
\end{tabular}

charge/discharge rates are $150 \mathrm{kWh}$ and $100 \mathrm{~kW}$, respectively. These values are considered based on the system nominal load in [3]. Similar to the previous example, the DFS-based cycle detection approach is employed to detect possible loops assuming that all the switches are closed and 1949 loops are detected.

1) Deterministic DSR: System nominal load in [3] is used for the deterministic optimization. The optimal system configuration and scheduled output power from batteries obtained by deterministic MIP are exhibited in Table VI. Table VI includes two calculated system loss: new configuration with and without battery units. The obtained topology reduces the active power loss by $18.4 \%$.

TABLE VI

DETERMINISTIC RECONFIGURATION 86-BUS SYSTEM

\begin{tabular}{|c|c|}
\hline Parameter & Result \\
\hline Optimal solution- open & $\begin{array}{c}12-61,13-76,14- \\
17,15-19,18- \\
34,20-24,20- \\
\text { switches } \\
36,23-25,39- \\
43,48-69,50- \\
51,52-85,70-86\end{array}$ \\
\hline $\begin{array}{c}\text { Total battery output } \\
(\mathrm{kW})\end{array}$ & 711.57 \\
\hline $\begin{array}{c}\text { Loss- configuration, } \\
\text { without battery }\end{array}$ & 1691.6 \\
\hline $\begin{array}{c}\text { Loss- new } \\
\text { configuration, with } \\
\text { battery (kW) }\end{array}$ & 1630.9 \\
\hline $\begin{array}{c}\text { Loss- original system } \\
(\mathrm{kW})\end{array}$ & 2070.7 \\
\hline
\end{tabular}

2) DSR with load uncertainty: The same load uncertainty characteristics explained in section III-A-2 are considered for this test system as well. Table VII shows the reconfiguration results obtained by SC FMIP, HC FMIP, and SMIP. Power flow is run for the results obtained by these algorithms (system topology and batteries scheduled output power) when the system nominal load is used. The calculated active power loss is shown in Table VII. HC FMIP and SMIP give the same configuration with minor differences in battery discharges. The results are tested on 5000 scenarios with different loads. As shown in Table VIII, HC FMIP and SMIP have a similar level of robustness (HC FMIP gives a slightly more robust solution with more power extracted from the batteries). Given the fact that HC FMIP is much faster than SMIP, it is recommended to use this approach for real-time applications.

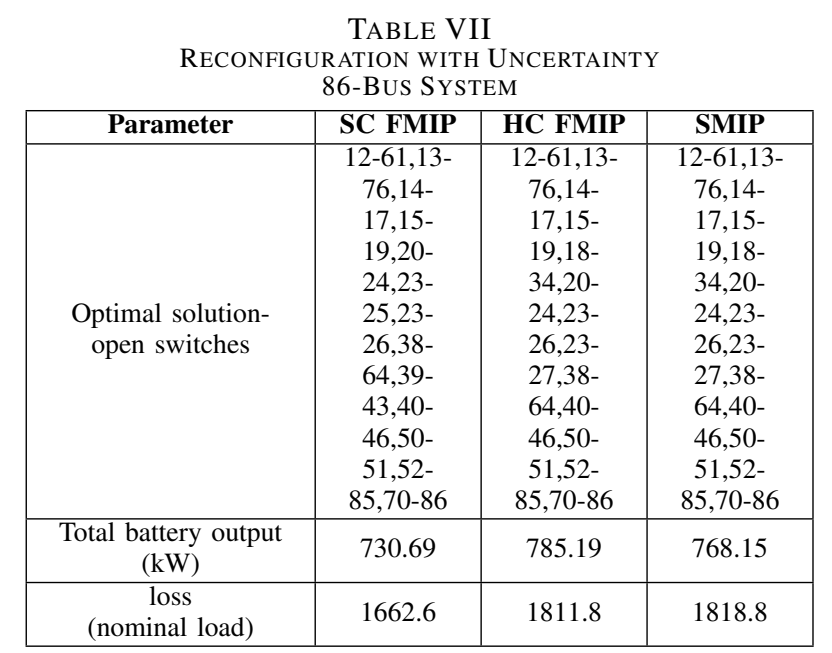

TABLE VIII

COMPARISON OF THE APPROACHES 86-BUS SYSTEM

\begin{tabular}{|c|c|c|c|c|}
\hline & Deterministic & SC FMIP & HC FMIP & SMIP \\
\hline $\begin{array}{c}\text { Infeasible } \\
\text { scenarios } \\
(\%)\end{array}$ & 35.6 & 12.7 & 3.3 & 3.34 \\
\hline $\begin{array}{c}\text { Computation } \\
\text { time (s) }\end{array}$ & 1.3 & \multicolumn{2}{|c|}{2.6} & 650 \\
\hline
\end{tabular}

\section{CONCLUSION}

This paper presents a robust MIP-based reconfiguration method to minimize loss in distribution systems. Radiality constraint is guaranteed by employing a DFS-based approach. This algorithm detects all the possible cycles assuming all the switches are closed in the system. Then, the optimization can guarantee the radiality of the distribution system by opening at least one switch in each loop. This leads to fast reconfiguration optimization even for large systems since the cycle detection approach is performed in off-line. Since distributed battery storage units are getting increased attention in distribution networks, mainly due to renewable integration and peak shaving purposes, this study analyzes the reconfiguration process assuming that there are battery storage units in the system. Due to insufficient measurements and increasing deployment of renewable resources and demand response programs in distribution grids, uncertainty in loads will be an important challenge for practical 


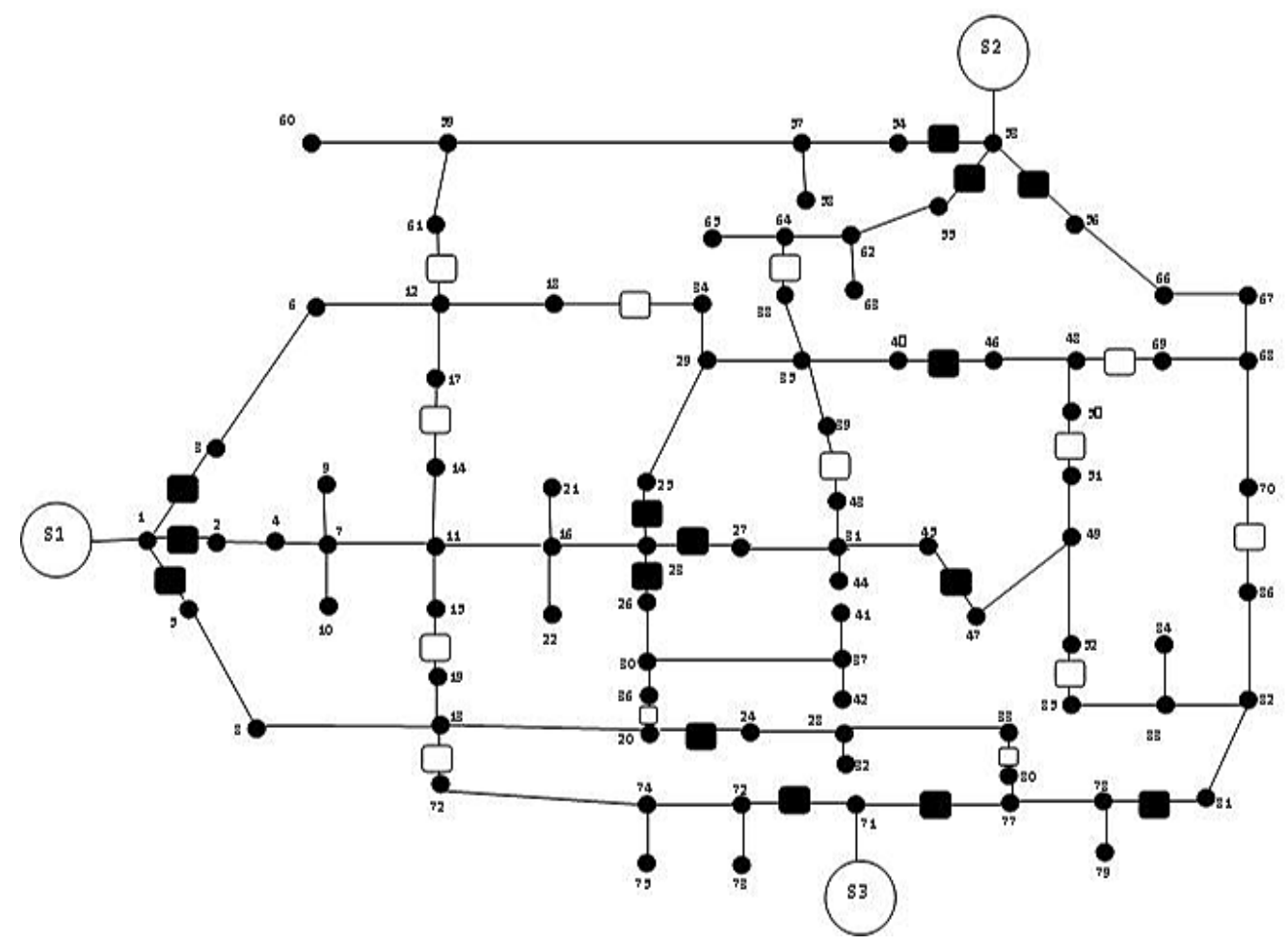

Figure 5. 86-bus test system

reliable distribution reconfiguration. Two different methods based on FMIP and SMIP are proposed to obtain robust topologies which are feasible for various load scenarios. It is shown that SMIP and FMIP both lead to more robust results, which are feasible for most possible load scenarios. The FMIP achieves nearly identical robustness with far less computation time and as such it may be preferable to use this approach for real-time applications. It is worth noting that the loss minimization DSR can also be expanded over a larger simulation period, e.g. 24 hours. In this case, the optimal configuration and battery scheduling (charging and discharging) will be calculated so that the total system loss will be minimized over 24 hours. This will be investigated in the authors' future work.

\section{REFERENCES}

[1] A. Abur, "A modified linear programming method for distribution system reconfiguration," Journal of Electric Power and Energy Systems, vol. 18, pp. 469-474, Oct. 1996.

[2] H. M. Khodr, J. MartÃnez-Crespo, M. A. Matos, and J. Pereira, "Distribution systems reconfiguration based on OPF using Benders Decomposition," IEEE Trans. on Power Delivery, vol. 24, pp. 2166- 2176, Oct. 2009.

[3] H. P. Schmidt, N. Ida, N. Kagan, and J. C. Guaraldo, "Fast reconfiguration of distribution systems considering loss minimization," IEEE Trans. on Power Systems, vol. 20, pp. 1311-1319, Aug. 2005.
[4] F. V. Gomes, S. Carneiro, J. L. R. Pereira, M. P. Vinagre, P. A. N. Garcia, and L. R. Araujo, "A new distribution system reconfiguration approach using optimum power flow and sensitivity analysis for loss reduction," IEEE Trans. Power Systems, vol. 21, no. 4, pp. 1616-1623, Nov. 2006.

[5] D. Shirmohammadi, and H Wayne Hong, "Reconfiguration of electric distribution networks for resistive line losses reduction," IEEE Trans. on Power Systems, vol. 4, pp. 1492-1498, Apr. 1989.

[6] G. K. V. Raju, and P. R. Bijwe, "An efficient algorithm for minimum loss reconfiguration of distribution system based on sensitivity and Heuristics," IEEE Trans. on Power Systems, vol. 23, pp. 1280-1287, Aug. 2008.

[7] Y.-T. Hsiao, "Multiobjective evolution programming method for feeder reconfiguration," IEEE Trans. on Power Systems, vol. 19, pp. 594-599, February 2004.

[8] A.Y. Abdelaziz, S.F. Mekhamer, F.M. Mohammed, and M.A.L. Badr, "A modified Particle Swarm Technique for distribution systems reconfiguration," Journal on Electronics and Electrical Engineering, vol.1, pp. 121-129, 2010.

[9] A. C. B. Delbem, A. C. de Carvalho, and N. G. Bretas, "Main chain representation for evolutionary algorithms applied to distribution system reconfiguration," IEEE Trans. Power Syst., vol. 20, no. 1, pp. 425-436, Feb. 2005.

[10] J. Mendoza, R. Lopez, D. Morales, E. Lopez, P. Dessante, and R. Moraga, "Minimal loss reconfiguration using genetic 
algorithms with restricted population and addressed operators: Real application,” IEEE Trans. Power Syst., vol. 21, no. 2, pp. 948-954, May 2006.

[11] J. Mendoza, R. Lopez, D. Morales, E. Lopez, P. Dessante, and R. Moraga, "Minimal loss reconfiguration using genetic algorithms with restricted population and addressed operators: Real application,” IEEE Trans. Power Syst., vol. 21, no. 2, pp. 948-954, May 2006.

[12] E. M. Carreo, R. Romero, and A. Padilha-Feltrin, "An efficient codification to solve distribution network reconfiguration for loss reduction problem," IEEE Trans. Power Syst., vol. 23, no. 4, pp. 1542-1551, Nov. 2008.

[13] M. A. Matos and P. Melo, "Loss minimization in distribution networks with multiple load scenarios," Proc. 2001 IEEE Power Tech, pp. 1-5.

[14] M. E. Baran, and F. F. Wu, "Network reconfiguration in distribution systems for loss reduction and load balancing," IEEE Trans. on Power Delivery, vol. 4, pp. 1401-1407. Apr. 1989.

[15] B. Moradzadeh, and K. Tomsovic, "Mixed integer programming-based reconfiguration of a distribution system with battery storage," Proc. 2012 North American Power Symposium (NAPS), pp. 1-6.

[16] M. Lavorato, J. F. Franco, M. J. Rider, and R. Romero, "Imposing radiality constraints in distribution system optimization problems," IEEE Trans. Power Systems, vol. 27, no. 1, pp. 172-180, Feb. 2012.

[17] P. Zhang, W. Li, and S. Wang, "Reliability-oriented distribution network reconfiguration considering uncertainties of data by interval analysis," Journal of Electrical Power and Energy Systems, vol. 34, pp. 138-144, Jan. 2012.

[18] D. S. Popovic, and Z. N. Popovic, "A risk management procedure for supply restoration in distribution network," IEEE Trans. on Power Systems, vol. 19, pp. 221-228, Feb. 2004.

[19] A. Kavousi-Fard, and T. Niknam, "Optimal distribution feeder reconfiguration for reliability improvement considering uncertainty," IEEE Trans. on Power Delivery, vol. 29, pp. 13441353, Jun. 2014.

[20] R. Arghandeh, and R. Broadwate, "Distributed Energy Storage Control for Optimal Adoption of Solar Energy in Residential Networks," Proc. 2012 American Society of Mechanical Engineers Power Conference, pp. 489-496.

[21] E. R. Ramos, A. G. Expsito, J. R. Santos, and F. L. Iborra, "Path-based distribution network modeling: application to reconfiguration for loss reduction," IEEE Trans. on Power Systems, vol. 20, pp. 556-564, May 2005.

[22] G. Liu and K. Tomsovic, "Robust Unit Commitment Considering Uncertain Demand Response," Electric Power Systems Research, vol. 119, pp. 126-137, Feb. 2015.

[23] B. Moradzadeh and K. Tomsovic, "Two-Stage Residential Energy Management Considering Network Operational Constraints," IEEE Trans. on Smart Grids, vol. 4, pp. 2339-2346, Dec. 2013.
[24] R. Bo and F. Li, "Probabilistic lmp forecasting considering load uncertainty," IEEE Trans. on Power Systems, vol. 24, pp. 1279-1289, Aug. 2009.

[25] L. Wu, M. Shahidehpour, and T. Li, "Stochastic SecurityConstrained Unit Commitment" IEEE Trans. on Power Systems, vol. 22, pp. 800-811, May 2007. 